\title{
Evaluation de schémas numériques pour la modélisation de l'advection-dispersion
}

\author{
L. Simon $(*)$
}

\section{Notations}

$C=$ Concentration en polluant $\left(\mathrm{kg} / \mathrm{m}^{3}\right)$

$C_{x}=\frac{\partial C}{\partial x}=$ dérivée première de la concentration suivant la direction $O_{x}$

$Q=$ Débit $\left(\mathrm{m}^{3} / \mathrm{s}\right)$

$A=$ Section mouillée rivière $\left(\mathrm{m}^{2}\right)$

$U=$ Vitesse moyenne dans la section $(\mathrm{m} / \mathrm{s})$

$Q=$ A.U.

$D=$ Coefficient de dispersion longitudinale $\left(\mathrm{m}^{2} / \mathrm{s}\right)$

$\Delta t=$ Pas de temps de la simulation

$\Delta x=$ Pas d'espace de la grille de calcul

$F_{i}^{n}=$ Valeur de la fonction $F$ au point d'abscisse $x_{i}=x_{1}+(i-1) \cdot \Delta x$ de la grille de calcul, au temps $t_{n}=t_{0}+n \cdot \Delta t, t_{0}=$ début de simulation.

Avertissement : On s'intéresse ici exclusivement à l'application de méthodes numériques à la résolution de l'équation d'advection-dispersion, laquelle décrit l'évolution des concentrations d'un composé dissous sous l'effet de l'hydrodynamique (à l'exclusion de toute réaction physico-chimique).

\section{Caractérisation des problèmes numériques : Nombres de Courant et de Péclet}

Lorsque l'on met en œuvre une méthode de résolution numérique, on se ramène tout d'abord au problème de résoudre les équations, continues, en des intervalles discrets d'espace et de temps. Ce faisant, on introduit des paramètres qui caractérisent le problème numérique à traiter, paramètres qui dépendent tout à la fois de spécificités physiques (champ de vitesses, coefficients de dispersion, ....) et de la façon de discrétiser.
Ces paramètres sont le nombre de Courant et le nombre de Péclet, qui s'expriment, dans le cas monodimensionnel :

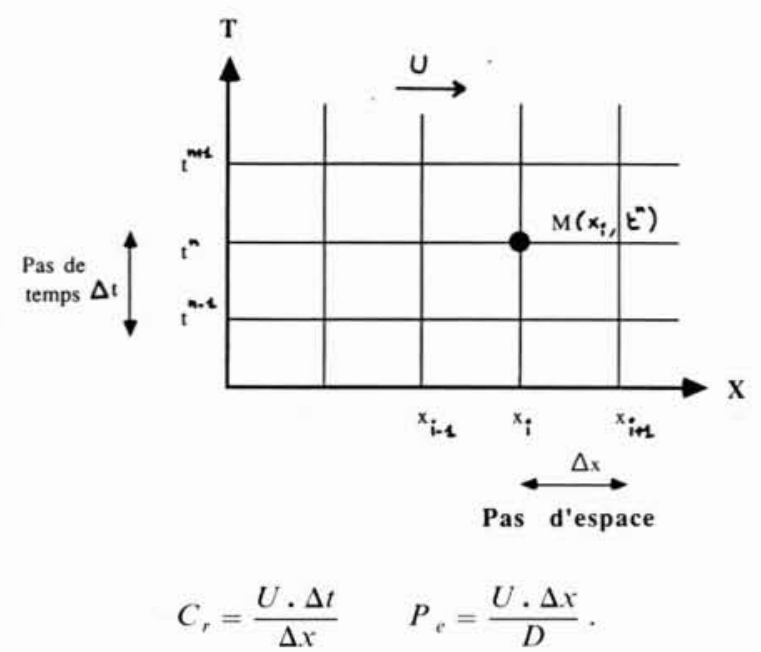

Le nombre de Courant "mesure" le nombre de mailles de la grille de calcul qu'une particule fluide parcourt en un pas de temps, le nombre de Péclet quantifie en quelque sorte l'importance relative des phénomènes d'advection et de dispersion, à l'échelle de la grille de calcul. Pour une diffusion nulle... ou des vitesses très élevées, on aura un Péclet élevé. Au contraire, quand la diffusion est importante, ou les vitesses faibles (ex.: lacs), la valeur du Péclet sera modérée.

(*) Centre d'enseignement et de recherche pour la gestion des ressources naturelles et de l'environnement (CERGRENEENPC), La Courtine, 93167 Noisy-le-Grand Cedex.

\section{Comparison of numerical schemes for the advection-dispersion equation}

To assess water quality after some transient events (accidental contamination, urban storm overflows, ...), one needs to take into account the hydrodynamic features of the receiving water body and the advection-dispersion phenomena. We present here two groups of numerical schemes for the solution of advection-dispersion equation and an evaluation of their applicability domain. 
$C_{r}$ et $P_{e}$ ne sont bien sûr pas uniformes dans un cas réel (variations spatio-temporelles de $U \ldots$..).

L'idéal serait bien sûr de discrétiser espace et temps uniquement en tenant compte des caractères physiques du problème étudié: pouvoir ainsi adopter de très grands pas d'espace dans les zones où l'on sait que les concentrations ne varient que fort peu le long de la rivière, adopter des pas de temps conformes aux échelles de temps des phénomènes simulés, ou dépendant de la fréquence à laquelle on dispose d'informations sur les entrées (amont, rejets) du système étudié. Malheureusement, les contraintes de mise en œuvre des schémas rendent souvent cela impossible.

Quels sont, dans le milieu naturel, les ordres de grandeur des nombres de Courant et Péclet? Ils sont bien sûr fortement dépendants du site étudié. A titre d'exemple, nous citerons deux cas fort différents.

Le premier est celui de l'Arc à l'aval d'Aix-en-Provence [1], petite rivière au régime fortement influencé par le rejet de la station d'épuration d'Aix $(30 \%$ du débit d'étiage de $1 \mathrm{~m}^{3} / \mathrm{s}$ ).

Des données recueillies lors d'un traçage de juillet 1987 (étiage typique) nous ont permis de calculer les valeurs des nombres de Courant et de Péclet en différentes stations de la rivière, pour un pas d'espace de $100 \mathrm{~m}$ (ce qui donnerait environ 400 points de calcul entre Aix et l'Etang de Berre où se jette l'Arc) et un pas de temps de 10 min (l'étude de l'impact du cycle journalier de la station d'épuration demande des simulations sur 3 à 5 jours).

ARC.

\begin{tabular}{|l|c|c|c|c|}
\hline STATION & $\begin{array}{c}\mathrm{U} \\
(\mathrm{m} / \mathrm{s})\end{array}$ & $\begin{array}{c}\mathrm{D} \\
\left(\mathrm{m}^{2} / \mathrm{s}\right)\end{array}$ & $\mathrm{P}_{\mathrm{e}}(\Delta \mathrm{x}=100)$ & $\mathrm{C}_{\mathrm{r}}(\Delta \mathrm{x}=100,10 \mathrm{~min})$ \\
\hline PIOLINE & 0.27 & 1 & 27 & 1.6 \\
\hline MAURAN & 0.51 & 9.3 & 5.5 & 3 \\
\hline $\begin{array}{c}\text { St PONS } \\
1\end{array}$ & 0.7 & 2.5 & 2.8 & 4.2 \\
\hline $\begin{array}{c}\text { St PONS } \\
2\end{array}$ & 0.45 & 12.6 & 3.6 & 2.7 \\
\hline $\begin{array}{c}\text { St PONS } \\
3\end{array}$ & 0.25 & 3.5 & 7.1 & 1.5 \\
\hline
\end{tabular}

On note une forte hétérogénéité des nombres de Péclet et de Courant, qui peuvent atteindre des valeurs élevées (30 pour $P_{e}, 4$ pour $C_{r}$ ). Pour maintenir $C_{r}$ plus petit que 1 , on serait obligé, pour un pas d'espace de $100 \mathrm{~m}$, de travailler avec un pas de temps de l'ordre de $2 \mathrm{~min}$ !

Le deuxième exemple est celui de la Seine en amont de Paris, sur laquelle plusieurs campagnes de traçage ont été effectuées en 1981-1982 (Modèle DISPERSO,
Agence Financière de Bassin Seine-Normandie, Compagnie Générale des Eaux).

Nous appuyant sur les abaques «Débits-Vitesses de transfert " et "Vitesse-Diffusion longitudinale " établies à cette occasion, nous avons estimé les ordres de grandeur de $C$, et $P_{e}$ pour différents débits, toujours pour $\Delta x=100 \mathrm{~m}$ et $\Delta t=10 \mathrm{~min}$, valeurs qui semblent raisonnables vu la géométrie de la Seine (qui ne varie que lentement) et les durées de simulation en jeu en cas de pollution accidentelle.

\begin{tabular}{|c|c|c|c|c|}
\hline & \multicolumn{2}{|c|}{, } & SEINE & \\
\hline$\underset{\left(m^{3} / \mathrm{s}\right)}{Q}$ & $\underset{(\mathrm{m} / \mathrm{s})}{U_{M}}$ & $\underset{\left(\mathrm{m}^{2} / \mathrm{s}\right)}{\mathrm{D}}$ & $P_{e}(\Delta x=100)$ & $C_{r}(\Delta x=100,10 \mathrm{~min})$ \\
\hline 50 & 0.15 & 0.7 & 21.5 & 0.9 \\
\hline 100 & 0.27 & 2.5 & 11 & 1.6 \\
\hline 150 & 0.4 & 4.6 & 8 & 2.2 \\
\hline 300 & 0.6 & 11.4 & 5.4 & 3.6 \\
\hline 400 & 0.75 & 16.5 & 4.6 & 4.5 \\
\hline 700 & 1 & 30 & 3.5 & 6 \\
\hline
\end{tabular}

On note essentiellement une forte valeur des nombres de Courant. Là encore, une limitation sur le nombre de Courant imposerait de travailler avec des pas de temps faibles par rapport aux échelles de temps "naturelles » du problème physique.

\section{Description des schémas numériques}

Etant donné le grand nombre d'algorithmes proposés pour traiter l'advection-dispersion, le choix des schémas testés dans cette étude ne peut qu'être subjectif. Les méthodes sélectionnées l'ont été pour l'une ou l'autre des raisons suivantes :

- leur simplicité de mise en œuvre,

- leur représentativité par rapport à une classe de méthodes numériques,

- leur acuité reconnue.

Le traitement numérique des différents termes qui composent l'équation d'advection-dispersion est de difficulté inégale. En particulier les termes de diffusion posent peu de problèmes et sont généralement traités suivant la méthode proposée par CRANK-NICOLSON.

Les schémas testés ici se distinguent par la manière dont sont pris en compte les termes advectifs, 4 schémas utilisant en particulier la méthode des caractéristiques. 


\subsection{Différences finies}

Le principe des méthodes aux différences finies est :

- de ne calculer la valeur de la fonction concentration qu'en des points discrets de l'espace-temps $\left(x_{i}, t^{\prime \prime}\right)$;

- d'approximer les dérivées partielles de l'équation d'advection-dispersion, en un nœud $M\left(x_{i}, t^{\prime \prime}\right)$, par des différences faisant intervenir la concentration $C_{i}^{n}$ et les concentrations aux points de la grille voisins de $M$; ce en se basant généralement sur les développements en série de Taylor.

Nous nous sommes volontairement restreints à des méthodes ne faisant intervenir qu'une approximation d'ordre assez bas (second ou troisième ordre) des termes advectifs $(\partial c / \partial x)$, ce qui limite leur complexité numérique.

Pour les 4 méthodes retenues on se ramène, au pire, à des systèmes d'équations linéaires tridiagonaux, pour lesquels on dispose d'algorithmes de résolution extrêmement performants.

Nous ne rappellerons pas ici le développement complet des schémas testés. Nous renvoyons pour cela le lecteur aux références [2] (méthodes Mac Cormack et Briley-Mac Donald), [3, 4] (méthode Quick), [5] (méthode de Takacs), ou, pour une synthèse, à la référence [6].

Nous nous contentons d'en rappeler les caractéristiques principales dans le tableau ci-contre :

Nota: Pour tous les schémas, la dérivée partielle par rapport au temps $\partial c / \partial t$ est approximée par

$$
\left(\frac{\partial c}{\partial t}\right)_{i}=\frac{C_{i}^{n+1}-C_{i}^{n}}{\Delta t} .
$$

\subsection{Méthodes aux caractéristiques}

Elles reposent sur l'approximation suivante: advection et diffusion, qui sont des processus physiques simultanés, sont traitées comme si elles se produisaient successivement à l'intérieur d'un pas de temps (méthodes à pas fractionnaires, cf. [13]).

L'équation d'advection-dispersion est ici traitée sous sa forme non conservative :

$$
\frac{\partial C}{\partial t}+U \frac{\partial C}{\partial x}=\frac{1}{A} \frac{\partial}{\partial x}\left(A D \frac{\partial C}{\partial x}\right) .
$$

La première étape consiste en la résolution de l'équation de transport pur. On recherche une solution intermédiaire $C^{*}$ telle que :

$$
\frac{C^{*}-C^{n}}{\Delta t}+U \frac{\partial C}{\partial x}=0 \quad\left(e_{1}\right) \text {. }
$$

Puis, dans un deuxième temps on calcule $C^{n+1}$ telle que :

$$
\frac{C^{n+1}-C^{*}}{\Delta t}=\frac{1}{A} \frac{\partial}{\partial x}\left(A D \frac{\partial C}{\partial x}\right) \quad\left(e_{2}\right)
$$

Ce par une méthode aux différences finies classique (Crank-Nicolson...).

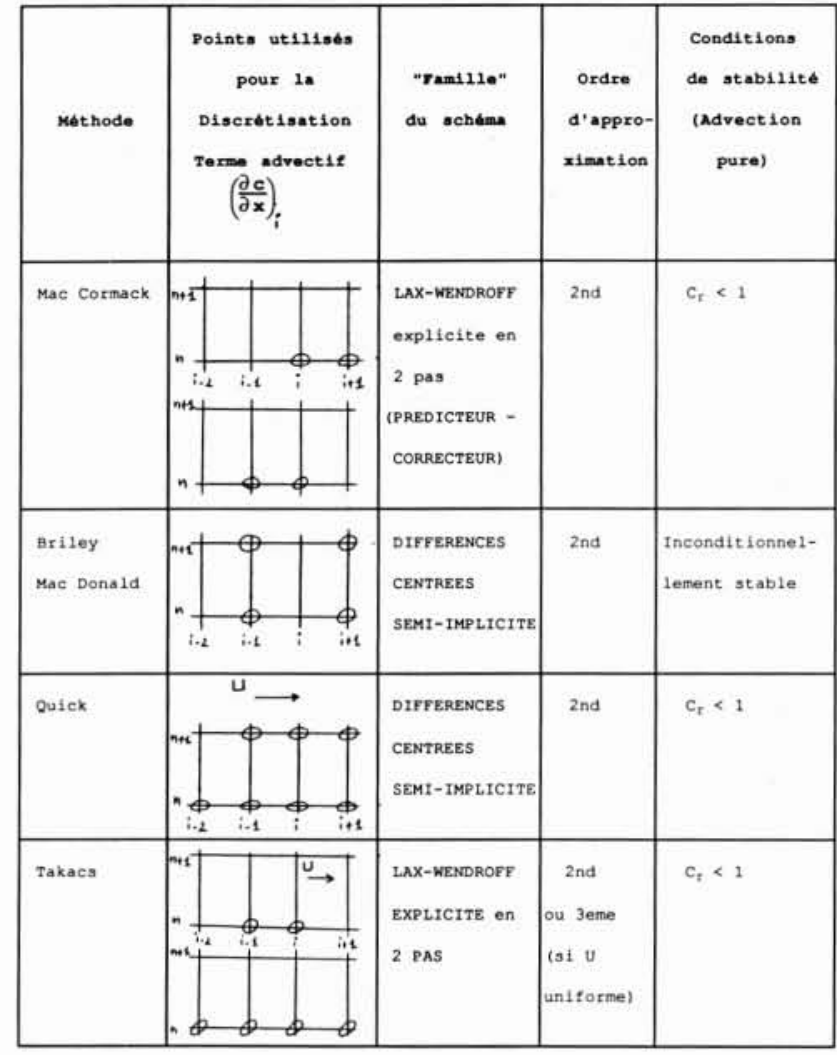

Les méthodes aux caractéristiques exploitent les propriétés des courbes caractéristiques de l'écoulement, qui sont les courbes tangentes au champ de vitesse.

Le long de ce type de courbe, d'équation $\frac{\mathrm{d} x}{\mathrm{~d} t}=U$, la dérivée totale de la concentration s'exprime en effet :

$$
\frac{\partial c}{\partial t}+U \frac{\partial c}{\partial x}\left(=\frac{\partial c}{\partial t}+\frac{\mathrm{d} x}{\mathrm{~d} t} \frac{\partial c}{\partial x}\right) .
$$

Donc, si la concentration vérifie une équation de transport pur, elle est constante le long d'une caractéristique.

Pour calculer $C^{*}$ au temps $t^{n+1}$ au point $M$ on va donc procéder comme suit :

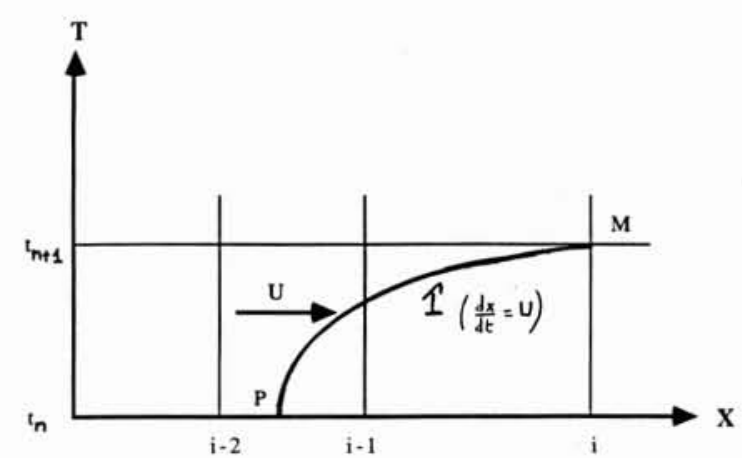

a) On recherche $P$ tel que la caractéristique passant par $M$ en $t^{n+1}$, passe par $P$ en $t^{n}$.

Pour cela il faut intégrer l'équation $\frac{\mathrm{d} x}{\mathrm{~d} t}=u(x, t)$ entre $t^{n}$ et $t^{n+1}$, avec pour condition limite $x\left(t^{n+1}\right)=M$. $M$. 
b) $P$ ne coïncide généralement pas avec un nœud de la grille de calcul.

Par suite, $C(P)$ doit être déterminée par interpolation d'après les différents $C_{j}^{n}$.
c) D'après
l'équation
de
transport,

$C^{*}(M)=C\left(P, t^{n}\right)$

Les différentes méthodes aux caractéristiques se distinguent essentiellement par la formule d'interpolation utilisée dans l'étape $(b)$. C'est de la qualité de celle-ci que dépendra l'acuité du schéma.

Pour déterminer la trajectoire, différentes méthodes sont possibles. Dans les applications numériques nous avons utilisé une méthode classique (Runge-Kutta du $4^{e}$ ordre), dont les détails peuvent par exemple être trouvés en [7]. Une erreur sur la détermination de $P$ aura le même effet que l'ajout d'un terme de dispersion parasite.

Pour les détails des interpolations et schémas testés, on se reportera à $[8,9]$ (HOLly-PreissmanN), [6] (Hop), [10] (RASCH-WILliamSON), [11] (DAN N'GUYEN), ou [6] pour une synthèse.

On se contente ici d'en rappeler les grands traits.

Pour les trois premières des méthodes citées ci-dessus, la concentration en $P$ est déterminée à partir des valeurs de la concentration et des dérivées spatiales de celle-ci aux nœuds qui encadrent $P$. Cependant,

- pour Holly-Preissmann, en dérivant l'équation d'advection-dispersion, on obtient une équation similaire qui régit les dérivées spatiales de la concentration ([8]), qu'on résout numériquement.

Cela conduit à travailler avec 2 variables, $C$ et $C_{x}$ ( 3 variables, $C, C_{x}, C_{y}$ dans le cas bidimensionnel) au lieu d'une seule $(C)$. Les calculs s'en trouvent alourdis. Par ailleurs, il faudra aussi imposer des conditions aux limites sur les dérivées, ce qui peut se révéler délicat! - pour Hop, version dégradée d'Holly-Preissmann, on estime les dérivées spatiales par différentiation centrée du champ de concentrations. On ne résout donc pas de nouvelle équation pour les obtenir ;

- pour Rasch-Williamson, on estime les dérivées spatiales à partir du champ de concentrations par une formule d'interpolation non linéaire (formule d'Akima).

La formule de Dan $N^{\prime}$ Guyen résulte d'un principe différent :

- on calcule d'abord deux estimations de la concentration en $P$, par développement de Taylor au second degré, l'un à partir du nœud en amont de $P$, l'autre à partir du nœud en aval de $P$;

- on pondère ces deux estimations.

Pour la pondération que nous avons sélectionnée, les schémas de Dan N'Guyen et Takacs sont équivalents pour un champ de vitesses uniforme, et un nombre de Courant inférieur à 1 .

\section{Domaines d'applicabilité de méthodes numériques}

En plus de la classique analyse de Fourier (cf. [6]), nous avons aussi appliqué aux schémas une batterie de tests numériques, nous appuyant sur les tests de référence établis par un groupe de travail international ([12]). Pour le détail de toutes les simulations effectuées et de leurs résultats, nous renvoyons à [6]. Nous nous contenterons ici d'illustrer d'un seul exemple les mérites et limites de chaque schéma, ou famille de schémas, sachant que les autres tests en ont confirmé les conclusions.

\subsection{Description du test sélectionné pour la présentation des performances numériques}

Le problème est gouverné par l'équation $(U, D$ constants) :

$$
\frac{\partial C}{\partial t}+U \frac{\partial C}{\partial x}=D \frac{\partial^{2} C}{\partial x^{2}} \quad(-\infty<x<\infty) .
$$

Conditions initiales: distribution gaussienne centrée en $x_{0}$, d'écart type $\sigma_{0}$, de concentration maximale 1 .

Conditions aux limites: $C(x, t) \rightarrow 0$ quand $|x| \rightarrow+\infty$.

Solution exacte au temps $t$ : distribution gaussienne centrée en $\bar{x}$, d'écart type $\sigma$, de concentration maximale $\sigma_{0} / \sigma$

$$
\bar{x}=x_{0}+U \cdot t \quad \sigma^{2}=\sigma_{0}^{2}+2 D t .
$$

Pour déterminer les conditions initiales $\left(\sigma_{0}\right)$, la grille de calcul et la durée de simulation $\left(T_{\max }\right)$ nous nous sommes inspirés des ordres de grandeur indiqués en [12]. Conditions du test

- $U=0,5 \mathrm{~m} / \mathrm{s}, \Delta x=200 \mathrm{~m}, T_{\max }=10800 \mathrm{~s}$ (la gaussienne parcourt $5,4 \mathrm{~km}$ )

- Gaussienne initiale : $\sigma_{0}=264 \mathrm{~m}=1,32 \Delta x$, $\rightarrow$ distribution initiale donnée à peu près par 7 points

- $C$, compris entre 0,12 à 1 (de 225 à 27 itérations jusqu'à $T_{\max }$ ), puis, uniquement pour les méthodes aux caractéristiques, de 1 à 3 ( 9 itérations)

- $P e:\{1,2,5,10,20,50,100,200,500,1000$, $\infty\}$.

\subsection{Résultats}

Pour analyser les performances des schémas, on utilise des mesures d'erreur (voir leurs définitions dans [12]), portant en particulier sur la conservation de la masse, sur le décalage induit par le schéma entre les positions des centres de gravité et pics de pollution des distributions calculée et exacte, sur la diffusion numérique du schéma ("étalement» artificiel du nuage de polluant), sur l'atténuation induite $(\varepsilon$, erreur relative sur la valeur des pics de concentration), les oscillations parasites (manifestations d'instabilité numérique, elles sont évaluées via le rapport $\varphi$ du maxima des concentrations négatives générées par le schéma sur la concentration maximale).

- Les schémas sont tous bien conservatifs. C'est particulièrement remarquable pour les schémas aux caractéristiques: la qualité de l'interpolation de l'étape d'advection compense la formulation non conservative du problème.

- C'est l'acuité du traitement de l'étape de diffusion qui conditionne la qualité du résultat pour les très faibles 
nombres de Péclet. On enregistre alors les plus petites erreurs pour les pas de temps les plus réduits, quel que soit le schéma.

- Dès que le phénomène advectif est important, les comportements divergent. Globalement, à Péclet donné, les performances des schémas aux différences finies classiques se dégradent quand le pas de temps augmente.

Le comportement des schémas aux caractéristiques est fondamentalement différent.

Quelle que soit la mesure d'erreur retenue, on a ici noté systématiquement une décroissance de l'erreur au fur et à mesure que le nombre d'itérations pratiquées pour atteindre $T_{\max }$, fin de la simulation, diminuait.

A chaque pas de temps, l'erreur est bien sûr fonction du nombre de Courant local (plus le pied de la caractéristique est proche d'un nœud de la grille de calcul, plus l'erreur d'interpolation est faible). Cependant, plutôt que de maintenir $C$, au voisinage d'un nombre entier, il apparaît plus efficace de tâcher globalement de réduire le nombre d'interpolations commises, donc d'augmenter le pas de temps (en restant toutefois dans les limites permises par la "physique " du problème).

- L'étude du décalage du pic de concentration est beaucoup plus instructive. Les schémas aux caractéristiques se montrent globalement beaucoup plus robustes (jamais d'erreur, par exemple, pour les schémas HollyPreissmann, Rasch-Williamson et Dan N'Guyen).

Pour les schémas aux différences finies, il n'existe qu'une gamme restreinte de nombres de Courant pour lesquels la position du pic de concentration est bien prévue (sauf pour Takacs, très robuste aussi).

Cette gamme est d'autant plus étroite que l'advection est importante (nécessité de nombres de courant très faibles pour Quick et Briley-Mac Donald, ou au contraire très proches de 1 pour Mac Cormack).

- Pour des Péclet forts, on observe parallèlement un étalement excessif du nuage de polluant et l'émergence de fortes concentrations négatives pour les schémas aux différences, et également pour les schémas Hop et Dan N'Guyen, mais d'une façon bien moins marquée (améliorations notables dès qu'on augmente le pas de temps).

- Pour exploiter les mesures $\varepsilon$ et $\varphi$, nous avons tracé, en fonction des nombres de Courant et de Péclet, les courbes suivantes :

- Isocontours correspondant à des erreurs relatives sur le pic de concentration de $5 \%, 10,15,20,25,30,35 \%$. - Isocontours correspondant à des concentrations négatives représentant respectivement 5 et $10 \%$ de la valeur du pic de concentration.

Le tracé de ces courbes, dont quatre exemples sont indiqués dans la figure ci-dessous, permet de cerner un domaine d'application des schémas, par exemple, selon le critère suivant :

" des concentrations négatives n'excédant pas $5 \%$ du maxima, un amortissement du maxima n'excédant pas $10 \% »$.

Comme l'illustre la figure, l'aire d'application des schémas aux caractéristiques excède toujours largement celle des schémas aux différences, et, particularité intéressante, ce domaine d'application est d'autant plus large que le pas de temps de la simulation est grand. Ainsi on peut envisager de traiter de façon satisfaisante même des problèmes fortement advectifs, ce qui est tout à fait exclu avec les schémas aux différences.
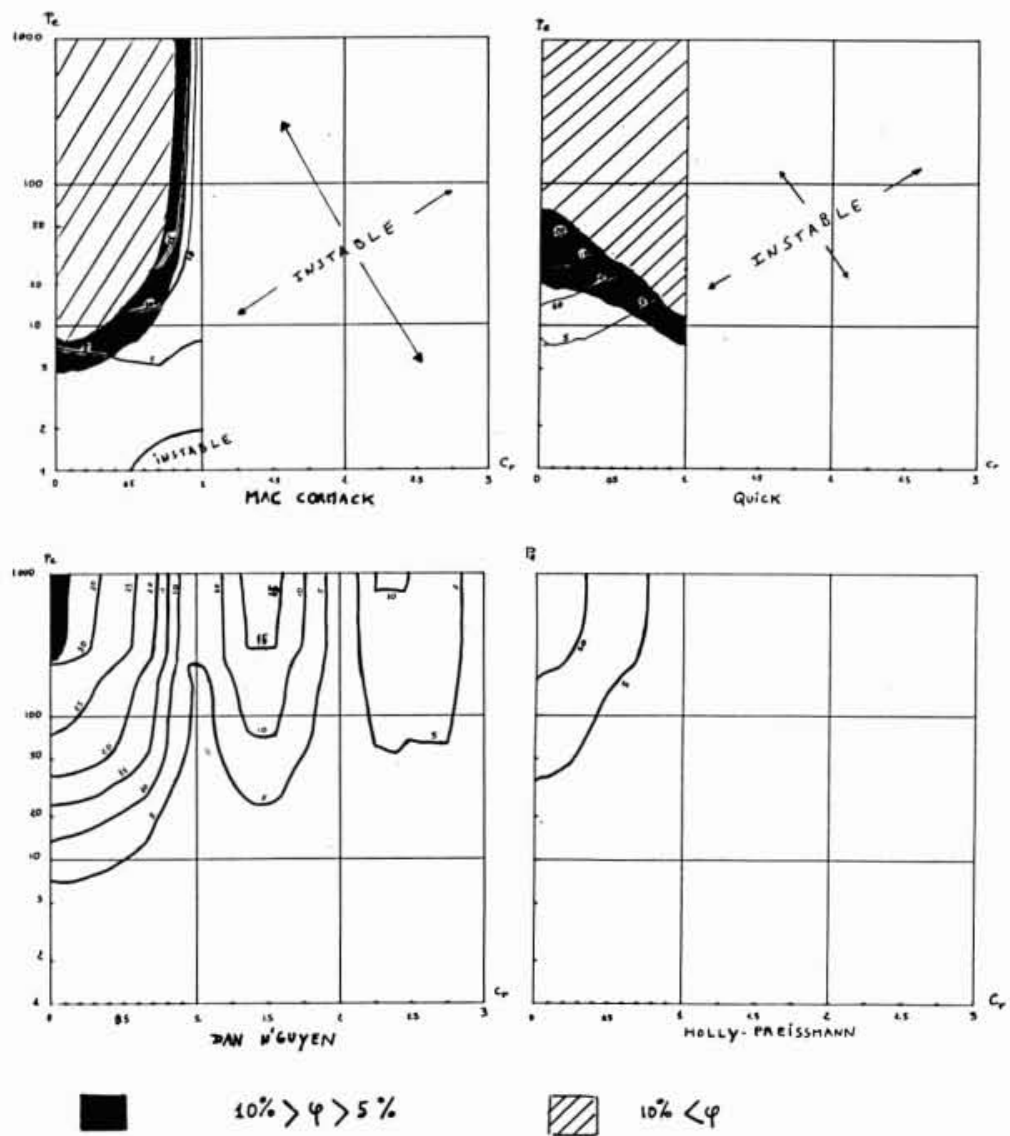


\subsection{Temps de calcul}

L'étude des temps de calcul fait apparaître que les schémas aux différences finies sont moins coûteux que les schémas aux caractéristiques. (NB : Tous les tests ont été effectués sur Vax 11/750). On observe par exemple pour le test bidimensionnel du Shearflow (cf. [12]) les rapports suivants entre les temps de calcul :

$$
\begin{array}{cccc}
\text { Holly-Pr } & \text { HOP, R-Will., Dan } & \text { Quick } & \text { Takacs } \\
1 & 0,5 & 0,36 & 0,25
\end{array}
$$

Ces coûts relatifs sont assez stables quel que soit le test pratiqué. Ainsi, les schémas aux différences sont de 25 à $50 \%$ moins chers que les schémas aux caractéristiques les plus simples, et le schéma le plus performant d'assez loin, celui d'Holly-Preissmann, est deux fois plus coûteux que les autres. Cependant, les schémas aux caractéristiques autorisent l'emploi d'un nombre de pas de temps plus faible par simulation. Au bout du compte, ils peuvent s'avérer aussi économiques que les schémas aux différences, sauf pour des champs de vitesse un peu complexes (nettes non-uniformité et non-stationnarité), où la recherche du pied de la caractéristique est coûteuse et peut représenter jusqu'à $90 \%$ du temps CPU (cf. [6]).

\section{Conclusions}

Les quelques résultats exposés ci-dessus n'ont pas pour objectif d'établir une hiérarchie entre quelques schémas mais plutôt d'illustrer la nécessité de tester à fond les outils numériques. Nous avons cherché à attirer l'attention sur quelques mesures d'erreur simples. En particulier, le tracé des isocontours d'erreurs sur les pics de concentration et des isocontours des concentrations négatives permet de visualiser les domaines dans lesquels on peut employer un schéma en confiance.

Il apparaît aussi nettement que les modélisateurs préoccupés par l'étude de phénomènes où l'advection est dominante se doivent d'abandonner les formulations classiques aux différences finies au profit des méthodes aux caractéristiques.

\section{Références}

[1] Mouchel. J. M., Zhang M. Z., Andre B., Bourrain X. — « Un modèle lagrangien de qualité. Application à l'Arc à l'aval d'Aix-en-Provence ". La Houille Blanche, ce numéro.

[2] Anderson, Tannehill et Pletcher. - "Computational Fluid Mechanics and Heat Transfer ”. Mac Graw Hill, 1984.

[3] LeONARD B. P. - "A stable and accurate modelling procedure based on quadratic upstream modelling". Computer Methods and Applied Mechanics in Engineering. vol. 19, p. 59-98, 1979.

[4] Falconer R. A., Suiquing Liu. - "Modelling solute transport using Quick scheme ». Journal of Environmental Engineering, vol. 114, $\mathrm{n}^{*} 1$, février 1988.

[5] TAKACS L. L. - «A two-step scheme for the advection equation with minimized dissipation and dispersion errors ". Monthly Weather Review, vol. 113, juin 1985.

[6] Simon L. - "Résolution numérique de l'équation d'advection-dispersion en mono et bidimensionnel ". Rapport interne CERGRENE, janvier 1990.

[7] WANG J. D. et al. - "Finite element characteristic advection model». Journal of Hydraulic Engineering. vol. 114, n* 9, septembre 1988.

[8] Holly F. M., Preissmann A. - " Accurate calculation of transport in two dimensions ". Journal of the Hydraulics Division, vol. 103, n* HY11, novembre 1977.

[9] Holly F. M., Usseglio-Polatera J. M. - « Dispersion simulation in two-dimensional tidal flow ". Journal of Hydraulic Engineering, vol. 110, n 7, juillet 1984.

[10] Williamson D. L., Rasch P. J. - «Semi-lagrangien transport with shape preserving interpolators". Proceedings Forum Convection-Diffusion, VIF International Conference on Computationnal Method in Water Resources, Cambridge, Massachusetts, U.S.A., juin 1988.

[11] DAN N'Guyen K. - "Modélisation numérique $2 D$ et $3 D$ de la circulation générale en milieux estuariens et côtiers ". Thèse de doctorat de l'Université de Paris VI, juin 1988.

[12] Baptista A., Gresho P., Adams E. - " reference problems for the 1988 Convection-Diffusion Forum n. $\mathrm{VII}$ International Conference on Computationnal Method in Water Resources, Cambridge, Massachusetts, U.S.A., juin 1988.

[13] YANENKo N. N. - "Méthode à pas fractionnaires". Armand Colin, collection intersciences, 1968.

Remerciements : Mes remerciements vont à Messieurs Usseglio-Polatera et Cunge, du L.H.F., qui m'ont communiqué l'énoncé des tests ainsi que certains éléments de la bibliographie, et à Monsieur Dan N'Guyen (Université de Lille, E.N.S. Montrouge) pour sa disponibilité. 